\title{
Used Cars Price Prediction using Supervised Learning Techniques
}

\author{
Pattabiraman Venkatasubbu, Mukkesh Ganesh
}

\begin{abstract}
The production of cars has been steadily increasing in the past decade, with over 70 million passenger cars being produced in the year 2016. This has given rise to the used car market, which on its own has become a booming industry. The recent advent of online portals has facilitated the need for both the customer and the seller to be better informed about the trends and patterns that determine the value of a used car in the market. Using Machine Learning Algorithms such as Lasso Regression, Multiple Regression and Regression trees, we will try to develop a statistical model which will be able to predict the price of a used car, based on previous consumer data and a given set of features. We will also be comparing the prediction accuracy of these models to determine the optimal one.
\end{abstract}

Keywords: ANOVA, Lasso Regression, Regression Tree, Tukey's Test

\section{INTRODUCTION}

$\mathrm{T}_{\mathrm{t}}$ The used car market is an ever-rising industry, which has almost doubled its market value in the last few years. The emergence of online portals such as CarDheko, Quikr, Carwale, Cars24, and many others has facilitated the need for both the customer and the seller to be better informed about the trends and patterns that determine the value of the used car in the market. Machine Learning algorithms can be used to predict the retail value of a car, based on a certain set of features.

Different websites have different algorithms to generate the retail price of the used cars, and hence there isn't a unified algorithm for determining the price. By training statistical models for predicting the prices, one can easily get a rough estimate of the price without actually entering the details into the desired website. The main objective of this paper is to use three different prediction models to predict the retail price of a used car and compare their levels of accuracy.

The data set used for the prediction models was created by Shonda Kuiper[1]. The data was collected from the 2005 Central Edition of the Kelly Blue Book and has 804 records of 2005 GM cars, whose retail prices have been calculated. The data set primarily comprises of categorical attributes along with two quantitative attributes.

The following are the variables used:

Revised Manuscript Received on December 02, 2019

* Correspondence Author

Pattabiraman Venkatasubbu*, School of Computing Science and Engineering, Vellore Institute of Technology, Chennai, India. Email: pattabiraman.v@vit.ac.in

Mukkesh Ganesh, School of Computing Science and Engineering, Vellore Institute of Technology, Chennai, India. Email: g.mukkesh2017@vitstudent.ac.in
Price: The calculated retail price of GM cars. The cars which were selected for this data set were all less than a year old and were considered to be in good condition.

Mileage: The total number of miles the car has been driven

Make: The manufacturer of the car

Model: The specific models for each car

Trim: The type of car model

Type: The car's body type

Cylinder: The number of cylinders present in the engine

Liter: The fuel capacity of the engine

Doors: The number of doors in the car

cruise: A categorical variable (binary), which represents whether cruise control is present in the car (coded 1 if present) sound: A categorical variable (binary), that represents whether upgraded speakers are present in the car (coded 1 if present)

Leather: A categorical variable (binary), that represents whether the car has leather interiors (coded 1 if present)

Using these attributes, we will try to predict the price by using the Statistical Analysis System (SAS) for exploratory data analysis.

\section{LITERATURE SURVEY}

Overfitting and underfitting come into picture when we create our statistical models. The models might be too biased to the training data and might not perform well on the test data set. This is called overfitting. Likewise, the models might not take into consideration all the variance present in the population and perform poorly on a test data set. This is called underfitting. A perfect balance needs to be achieved between these two, which leads to the concept of Bias-Variance tradeoff. Pierre Geurts [2] has introduced and explained how bias-variance tradeoff is achieved in both regression and classification. The selection of variables/attribute plays a vital role in influencing both the bias and variance of the statistical model. Robert Tibshirani [3] proposed a new method called Lasso, which minimizes the residual sum of squares. This returns a subset of attributes which need to be included in multiple regression to get the minimal error rate. Similarly, decision trees suffer from overfitting if they are not pruned/shrunk. Trevor Hastie and Daryl Pregibon [4] have explained the concept of pruning in their research paper. Moreover, hypothesis testing using ANOVA is needed to verify whether the different groups of errors really differ from each other. This is explained by TK Kim and Tae Kyun in their paper [5]. A Post-Hoc test needs to be performed along with ANOVA if the number of groups exceeds two. 


\section{Used Cars Price Prediction using Supervised Learning Techniques}

Tukey's Test has been explored by Haynes W. in his train and test the effectiveness of our statistical models.

\section{PROPOSED MODEL}

\section{A. Null Hypothesis}

Even though the magnitude of overfitting has been reduced, Regression trees still suffer from overfitting even after

Hypothesis: Multiple and Lasso Regressions are better at predicting price than the Regression Tree.

\section{B. Training and Testing Data}

The data is split into training(70\% - 563 records) and testing $(30 \%$ - 241 records) data sets through random sampling (seed was set to 2786).

\section{Lasso Regression}

Using Lasso regression on the training data set, we first select the subset of attributes which lead to optimal/least sum of squared error while predicting the price. It makes use of 10 -fold cross-validation to "lasso" the optimal subset of attributes. It uses L1 regularization.

\section{Table - 1: Lasso Regression Summary}

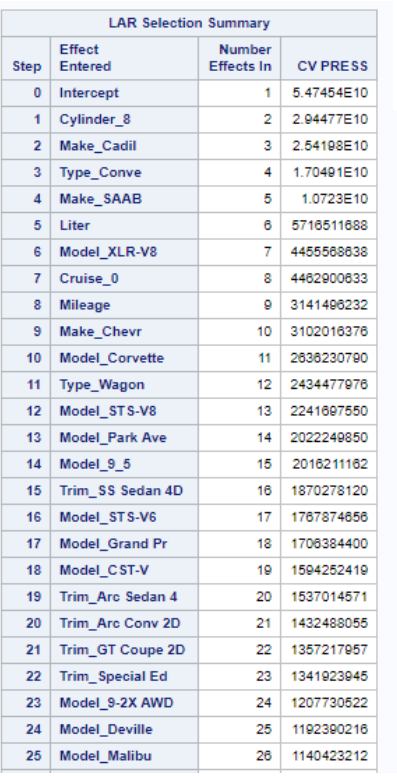

\begin{tabular}{|c|c|c|c|}
\hline 26 & Model_Lacrosse & 27 & 1079213317 \\
\hline 27 & Model_Vibe & 28 & 1057127900 \\
\hline 28 & Trim_SS Coupe 2D & 28 & 991121705 \\
\hline 29 & Trim_SVM Hatchba & 30 & 968461697 \\
\hline 30 & Trim_Sedan 4D & 31 & 906490734 \\
\hline 31 & Model_Cavalier & 32 & 800413251 \\
\hline 32 & Model_AVEO & 33 & 895244688 \\
\hline 33 & Trim_CXS Sedan 4 & 34 & 888133715 \\
\hline 34 & Model_Sunfire & 35 & 868698872 \\
\hline 35 & Trim_Custom Seda & 36 & 849386378 \\
\hline 36 & Trim_SVM Sedan 4 & 37 & 842938940 \\
\hline 37 & Model_Grand Am & 38 & 834462358 \\
\hline 38 & Trim_LS Coupe 2D & 39 & 820309420 \\
\hline 39 & Trim_LT Coupe 2D & 40 & 800493685 \\
\hline 40 & Trim_GXP Sedan 4 & 41 & 785550308 \\
\hline 41 & Model_Century & 42 & 780838005 \\
\hline 42 & Model_L Series & 43 & 757522044 \\
\hline 43 & Model_G6 & 44 & 734553436 \\
\hline 44 & Trim_GTP Sedan 4 & 45 & 708162714 \\
\hline 45 & Trim_Limited Sed & 46 & 691701586 \\
\hline 46 & Trim_AWD Sportwa & 47 & 687992677 \\
\hline 47 & Trim_CXL_Sedan 4 & 48 & 680838392 \\
\hline 48 & Trim_DTS Sedan 4 & 49 & 674240643 \\
\hline 49 & Leather_o & 50 & 664002260 \\
\hline 50 & Trim_Arc Wagon 4 & 51 & 662795833 \\
\hline
\end{tabular}

\begin{tabular}{|c|c|c|c|}
\hline 51 & Trim_DHS Sedan 4 & 52 & \\
\hline 52 & Trim_GT Sportwag & 53 & 6123060627 \\
\hline 53 & Make_ Satur & 54 & 8006677882 \\
\hline 54 & Trim_LS Sport Co & 55 & 6008009142 \\
\hline 55 & Model_Classic & 56 & 604819404 \\
\hline 56 & Trim_SLE Sedan 4 & 57 & 60101078887 \\
\hline 57 & Sound 0 & 58 & 509479062 \\
\hline 58 & Trim_GT Sedan 4D & 50 & 597018312 \\
\hline 59 & Trim_Linear Conv & 00 & 509172036 \\
\hline 60 & Trim_LT Sedan AD & 61 & 599797402 \\
\hline 61 & Trim_Coupe 2D & 82 & 505444703 \\
\hline 62 & Trim_Conv 2D & $\theta 3$ & 587887750 \\
\hline 63 & Trim_LT MAXX Hba & 64 & 580000103 \\
\hline 64 & Model_9_5 HO & 05 & 505774528 \\
\hline 65 & Trim_MAXXX Hback & 66 & 505584705 \\
\hline 66 & Trim_LS Sedan AD & 67 & 588109168 \\
\hline 67 & Model_Monte Ca & 68 & 5627455554- \\
\hline 68 & Trim_Quad Coupe & 68 & 5832000002 \\
\hline 69 & Trim_LT Hatohbac & 70 & 583400038 \\
\hline 70 & Trim_LS Hatchbac & 71 & $585911888 \mathrm{~B}$ \\
\hline 71 & Trim_Aero Wagon & 72 & 588112390 \\
\hline 72 & Trim_LS Sport Se & 73 & 5086820201 \\
\hline 73 & Trim_Aero Sedan & 74 & 5872008800 \\
\hline
\end{tabular}
research paper [6]. Using these techniques, we will create, Pruning. This leads to our following hypothesis.

The LAR Selection summary returns the levels of attributes which need to be chosen to reduce the prediction error.

We can infer from the table-1 that the cross-validated predicted residual error sum of squares (CV PRESS) is the least for the 67 levels of the chosen attributes. Fig. 1 gives us a graphical representation of this. All the chosen 12 attributes, except doors, were lassoed.

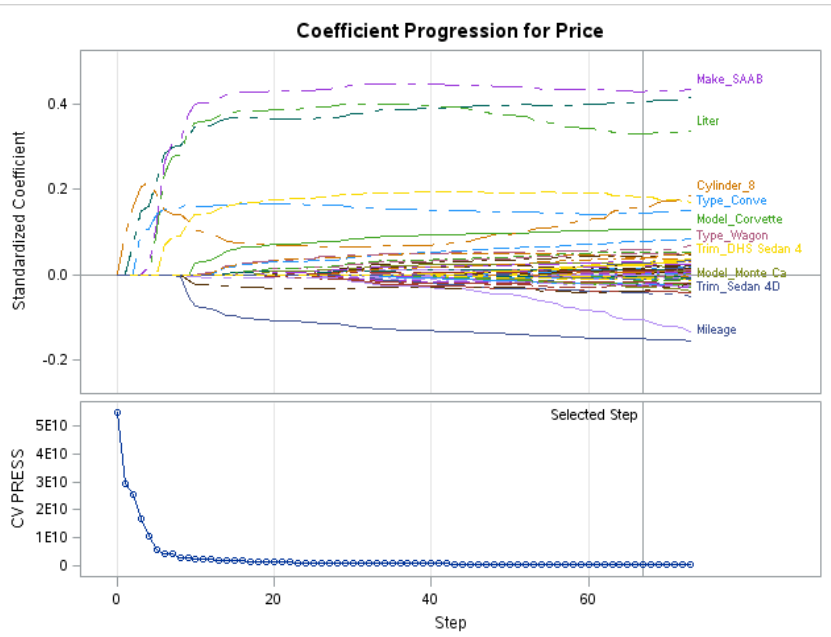

Fig. 1: The coefficients (estimates) of each parameter when other parameters are added is plotted. Also, the $\mathrm{CV}$-Press of the selection process is plotted.

The error rate reaches the minimum value when the above-mentioned levels of the variables were selected for multiple regression. This is shown in Figure 2.

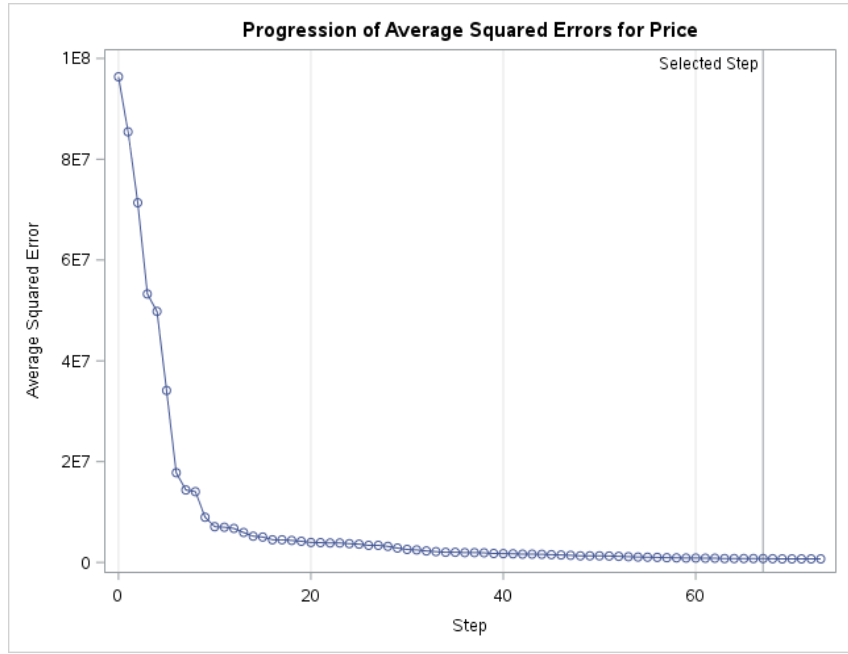

Fig. 2: The Average Square Error is also plotted against the number of levels of variables selected.

Since each level of the categorical variable is treated as a variable on its own (in multiple and Lasso regression), we get 67 estimates.

The prediction model works based on this generated equation:

Price $=$ Intercept $+P_{1} * E_{1}+P_{2} * E_{2}+\ldots \ldots+P_{67} * E_{67}$ 
Where $\mathrm{P}_{1}-\mathrm{P}_{67}$ are parameter values while $\mathrm{E}_{1}-\mathrm{E}_{67}$ are the parameter estimates. These parameter estimates are tabulated in Table 2.

\section{Table - 2: Parameter Estimates of Lasso Regression}

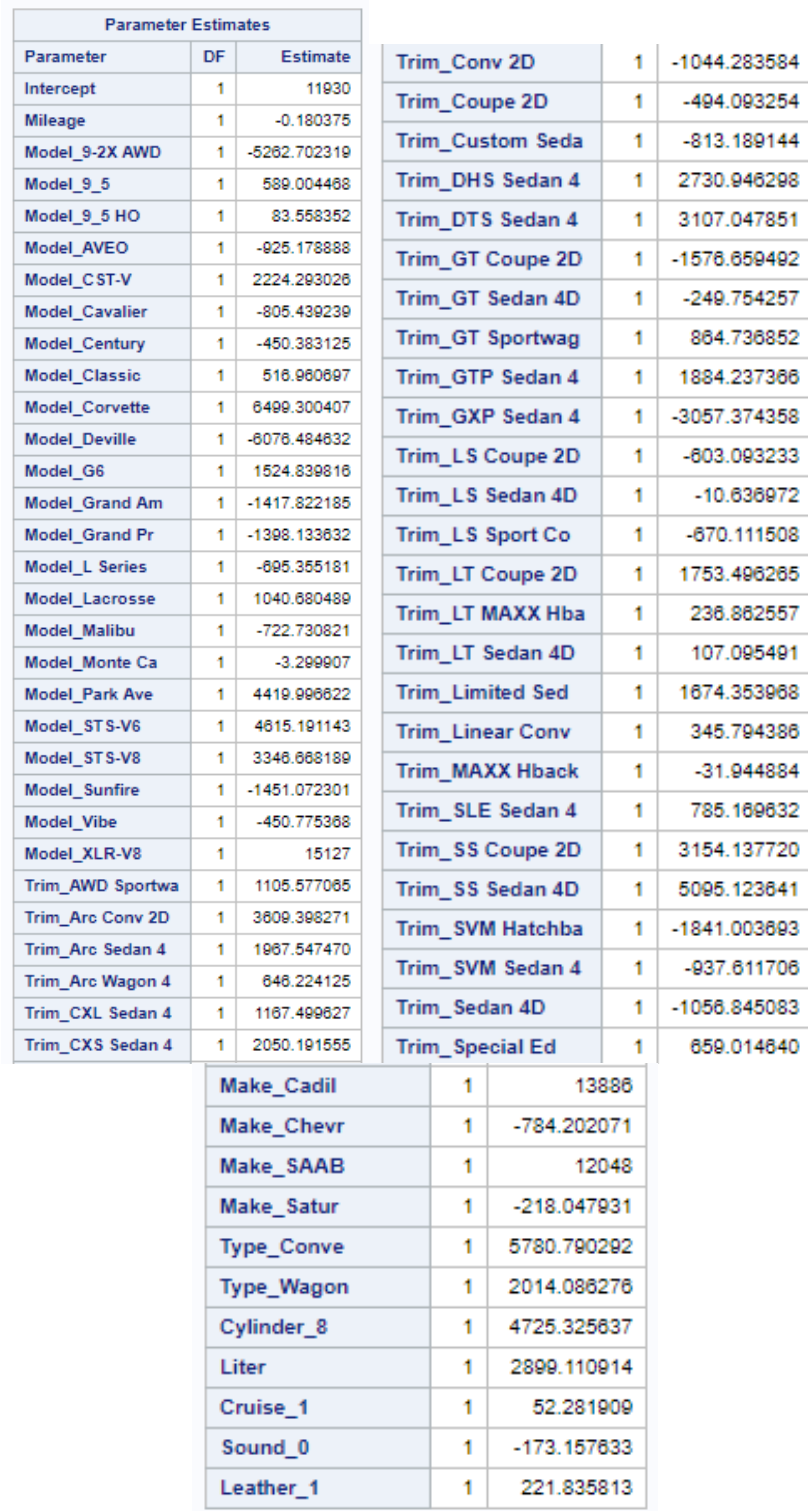

The parameter estimates of the 67 levels are tabulated here Since Lasso regression heavily relies on the training set to find the best fit levels of attributes, it might miss out on some levels of categorical variables which do not show much association in the training dataset, due to random sampling. This might cause our model to be slightly (maybe even statistically insignificant) underfit, since in-group variance might have been overlooked. Hence, an iterative process is needed to determine the mean error rate.

\section{Multiple Regression}

A general linear model, which models price to the set of selected attributes is trained (on the training data set). The results are tabulated in Table 3 . The variables which were selected in Lasso Regression are used here. However here, all the levels of the variables are taken into consideration.
Table - 3: Multiple Regression Summary

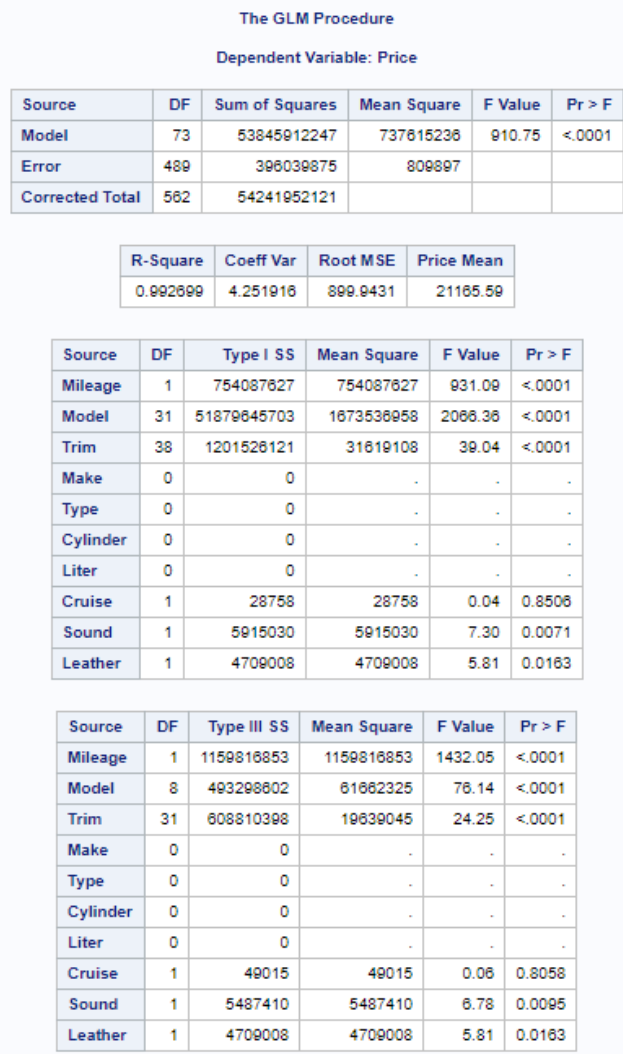

The result of the GLM procedure with $\mathrm{P}$-value and $\mathrm{R}^{2}$ values are tabulated along with the type 1 and type 3 error rates.

From this model, we can see that the variable Price and the selected variables are highly correlated since the R-Square (coefficient of determination) value is around 0.9927 . This implies that these variables account for about $99.27 \%$ of the variance in the Price.

Moreover, both Type 1 and Type 3 SS tables show us that all the variables are significantly correlated with Price $(\mathrm{P}$ values $<0.05$ ), except Cruise control, which is confounded when the other variables are held at their mean.

Similar to the GLM Select procedure, this procedure also returns a set of parameter estimates, for numerical variables and every level of the categorical variables.

Price $=$ Intercept $+P_{1} * E_{1}+P_{2} * E_{2}+\ldots \ldots+P_{n} * E_{n}$

Where $P_{1}-P_{n}$ are parameter values while $E_{1}-E_{n}$ are the parameter estimates. These parameter estimates are tabulated in Table 4. 


\section{Used Cars Price Prediction using Supervised Learning Techniques}

Table - 4: Parameter Estimates of Multiple Regression

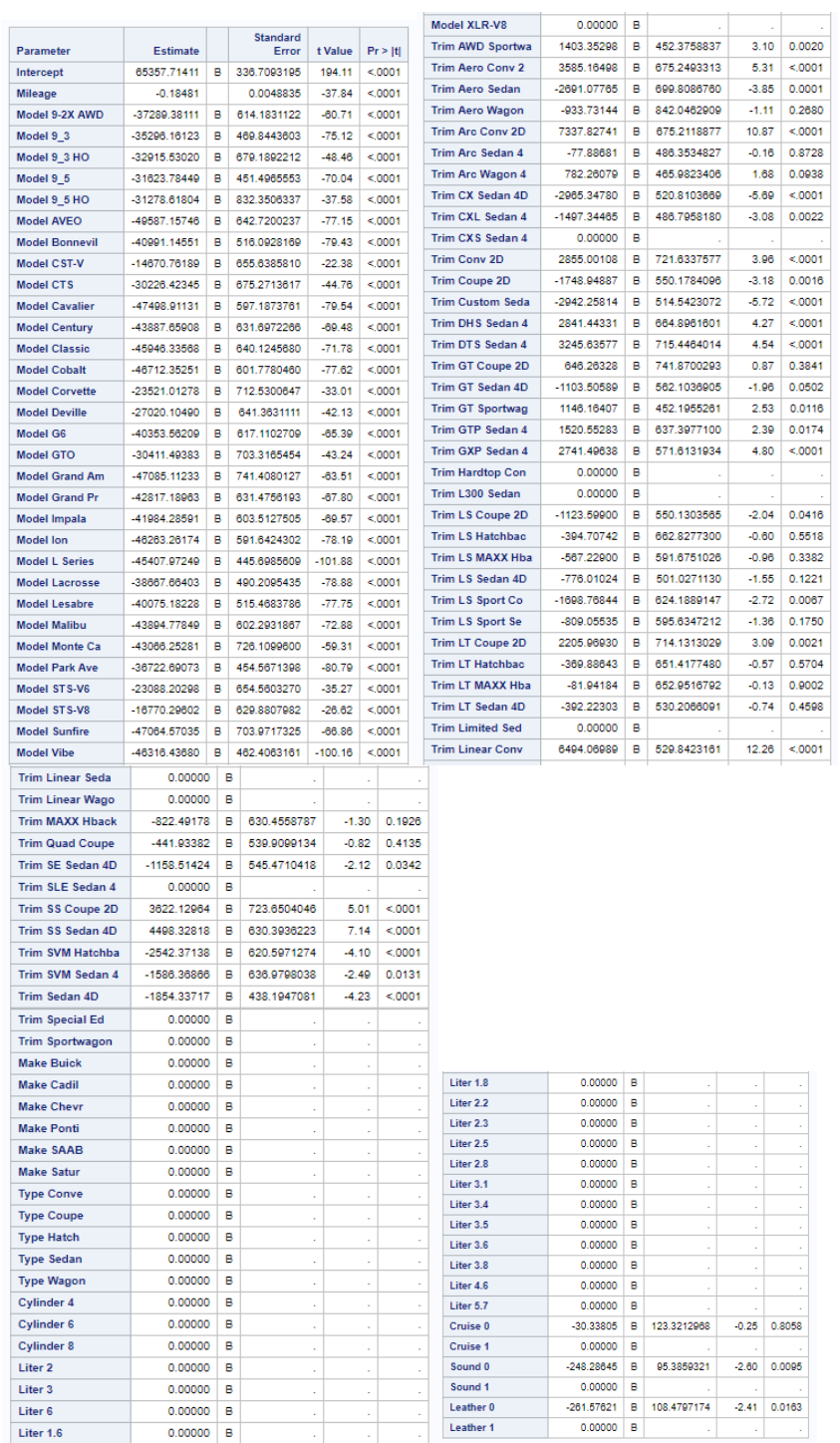

The parameter estimates of the 11 selected variables are tabulated here.

The QQ plot for the residual of the price (the difference between the observed and predicted values) and the histogram of the distribution of residuals show us that it approximately follows a normal distribution, with some outliers being present.

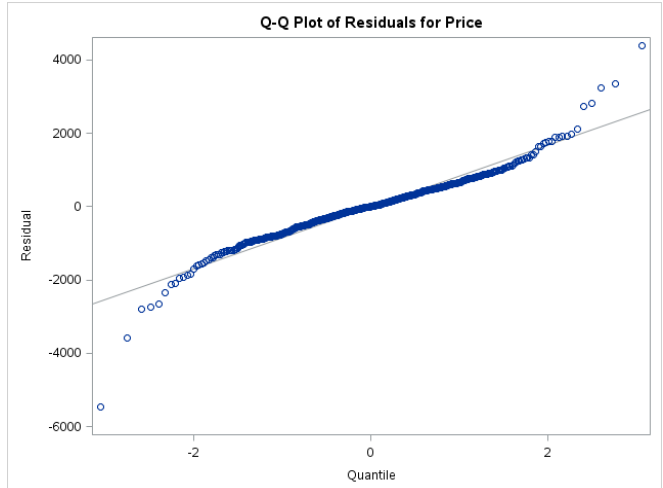

Fig. 3: The QQ-Plot of the residuals are plotted to check for normal distribution

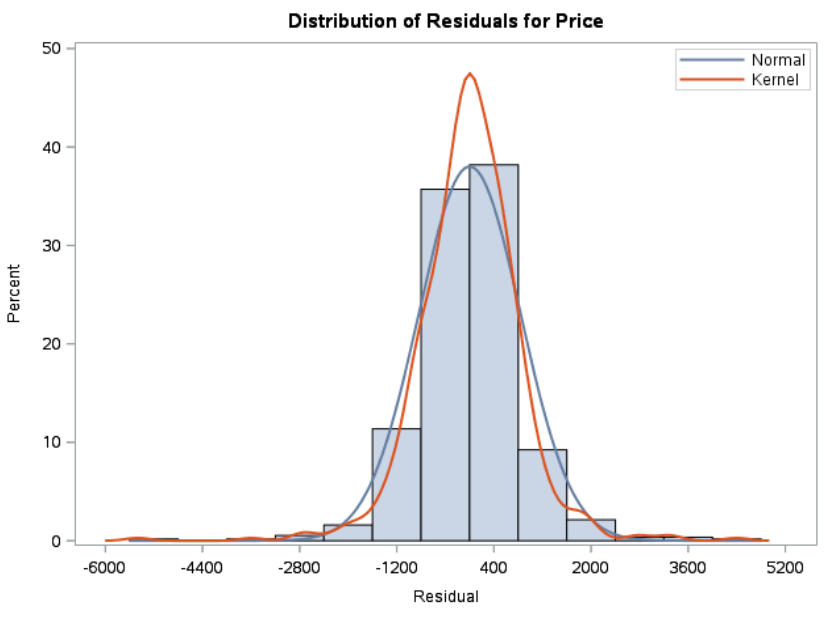

Figure 4: The distribution of residuals is plotted to check for normal distribution.

The studentized residual plot shows us the presence of around 28 outliers in this training data set.

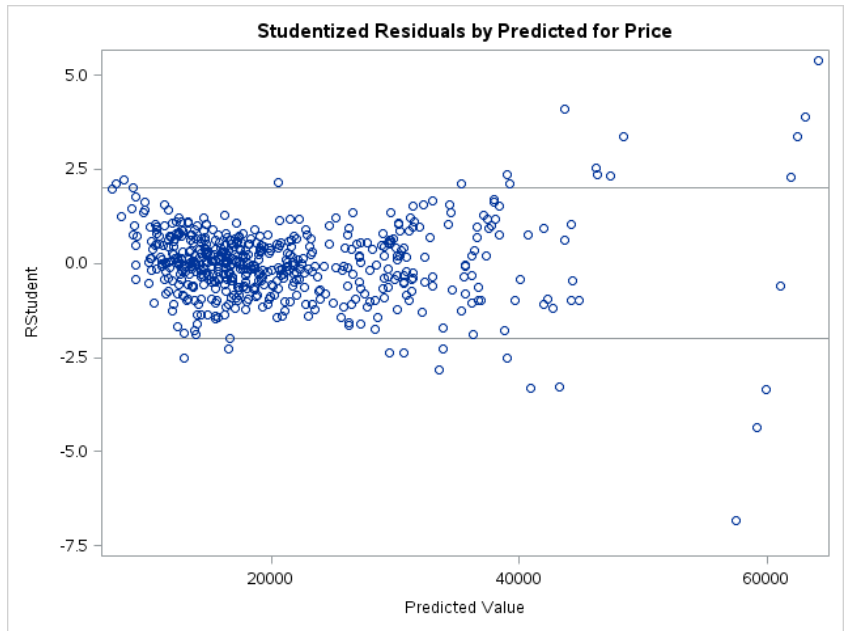

Figure 5: The Studentized residuals are plotted to check for outliers.

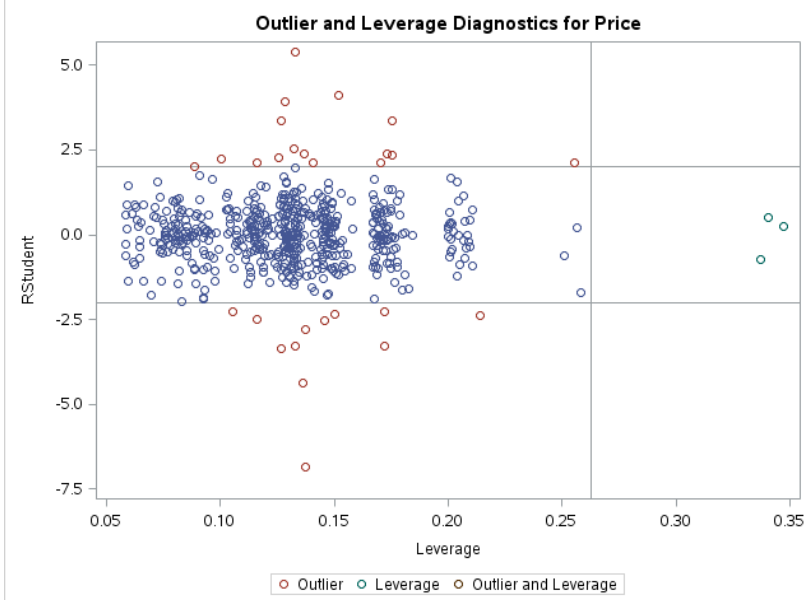

Figure 6: The studentized leverage and outlier plot is used to find whether there are any outliers which heavily influence the prediction model. 
The leverage and outlier plots show that these outliers do not hold any leverage. Hence, their absence from the data set doesn't affect the model significantly.

\section{E. Regression Tree}

A regression tree which models price to the selected subset of attributes is created (by using the training data set) by calling the HPSPLIT Procedure. The results are tabulated in table 5.

Table - 5: HPSPLIT Procedure summary

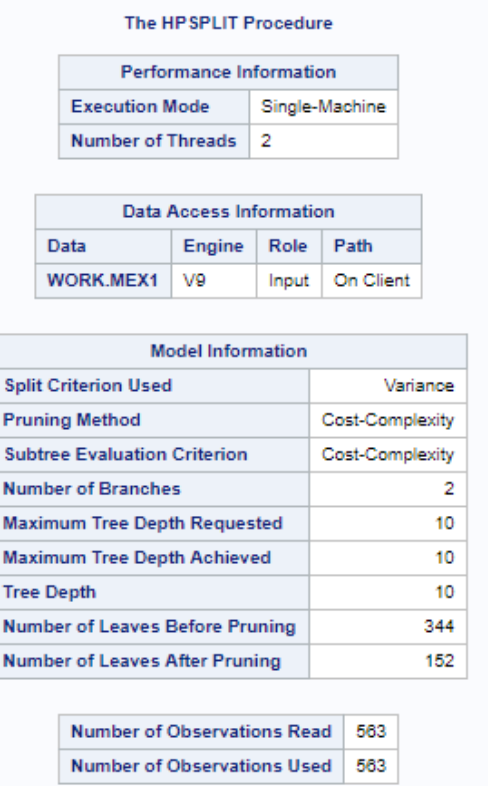

The HPSPLIT Procedure uses Variance for split criteria and Cost-Complexity for Pruning. The number of leaves before and after pruning is also shown.

This tree, before pruning, had 344 leaf nodes. Upon using the cost complexity algorithm for pruning, the number of leaves got reduced to 152 . The process of pruning is visually represented in figure 7 .

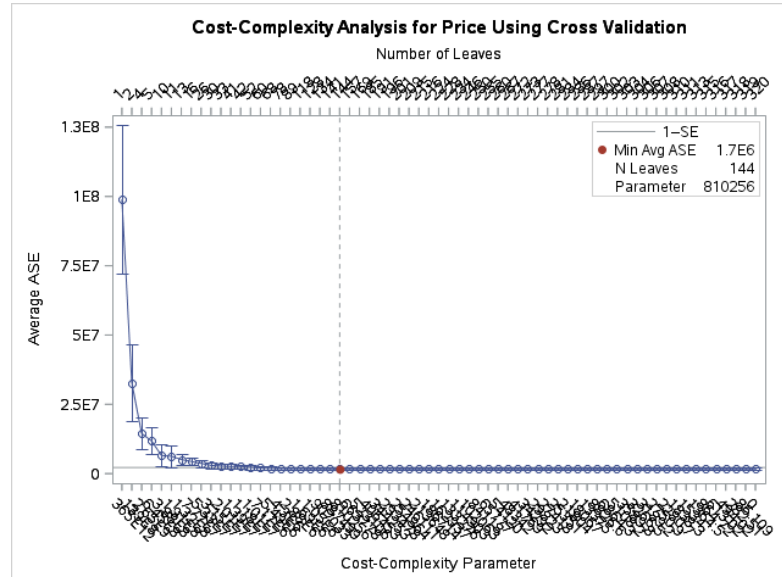

Figure 7: The Average Square Rate is plotted against the number of leaves and cost-complexity parameter to find the minimum ASE.

Here, the minimum average square error is 1.7E6, and that model is selected. The following tree (Fig 8) was produced,

which has reduced overfitting. The zoomed-in regression tree (Fig 9) is generated alongside the actual regression tree.

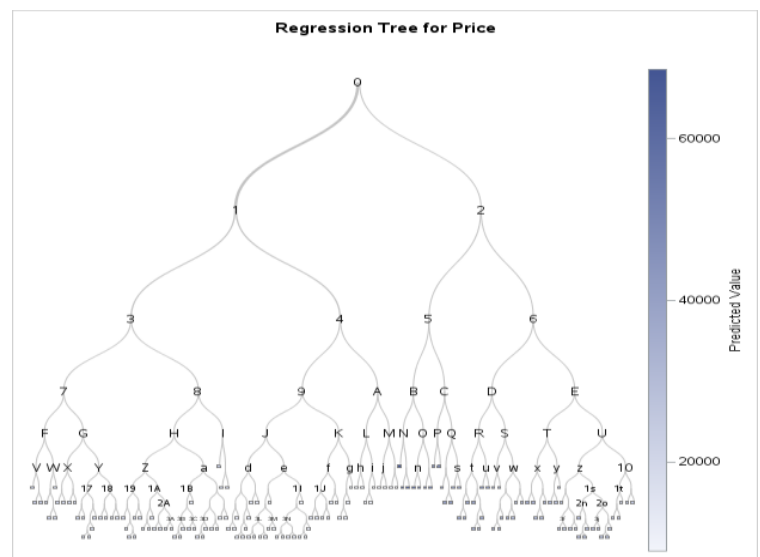

Figure 8: The Regression tree is graphically represented.

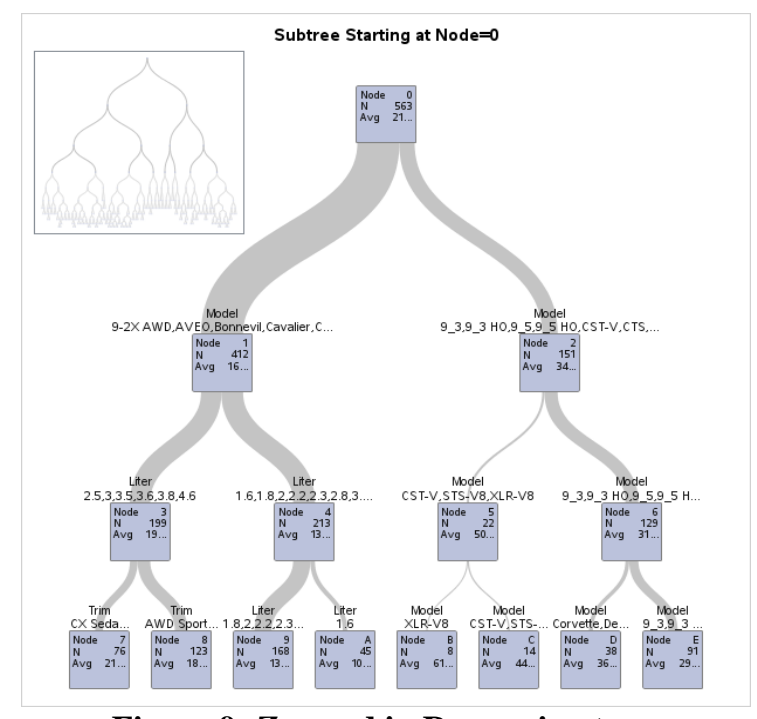

Figure 9: Zoomed in Regression tree.

The order of importance of the variables is also tabulated (table 6). From it, we can infer that the model of the car is most associated with price and that the presence/absence of upgraded sound systems is least associated with price.

Table - 6: HPSPLIT Attribute Importance

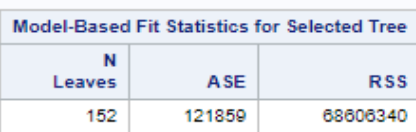

\begin{tabular}{|l|r|r|r|}
\hline \multirow{3}{|c|}{ Variable Importance } \\
\hline \multirow{2}{*}{ Variable } & \multicolumn{2}{|c|}{ Training } & \\
\cline { 2 - 3 } Model & Relative & Importance & Count \\
\hline Liter & 1.0000 & 215640 & 25 \\
\hline Trim & 0.3080 & 66418.3 & 5 \\
\hline Mileage & 0.1855 & 40004.7 & 31 \\
\hline Type & 0.1839 & 39651.1 & 80 \\
\hline Leather & 0.0410 & 8849.2 & 3 \\
\hline Make & 0.0106 & 2296.2 & 3 \\
\hline Sound & 0.0076 & 1629.4 & 2 \\
\hline
\end{tabular}




\section{Used Cars Price Prediction using Supervised Learning Techniques}

\section{F. Prediction on test data}

The 3 trained models were used to predict the price of the test data, which contained 241 records. The Observed vs Predicted graphs were plotted for all the three models.

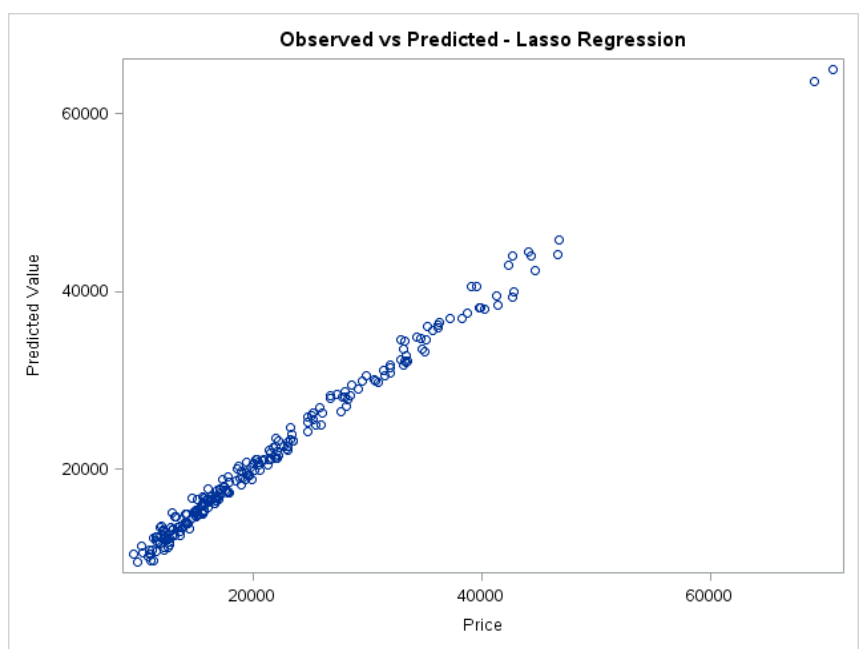

Figure 10: Observed vs Predicted Price - Lasso Regression.

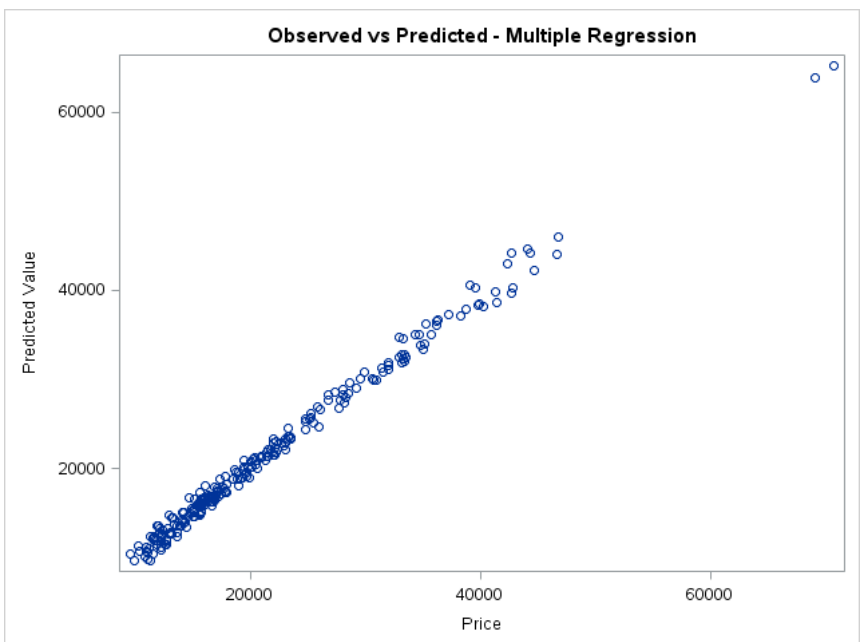

Figure 11: Observed vs Predicted Price - Multiple Regression.

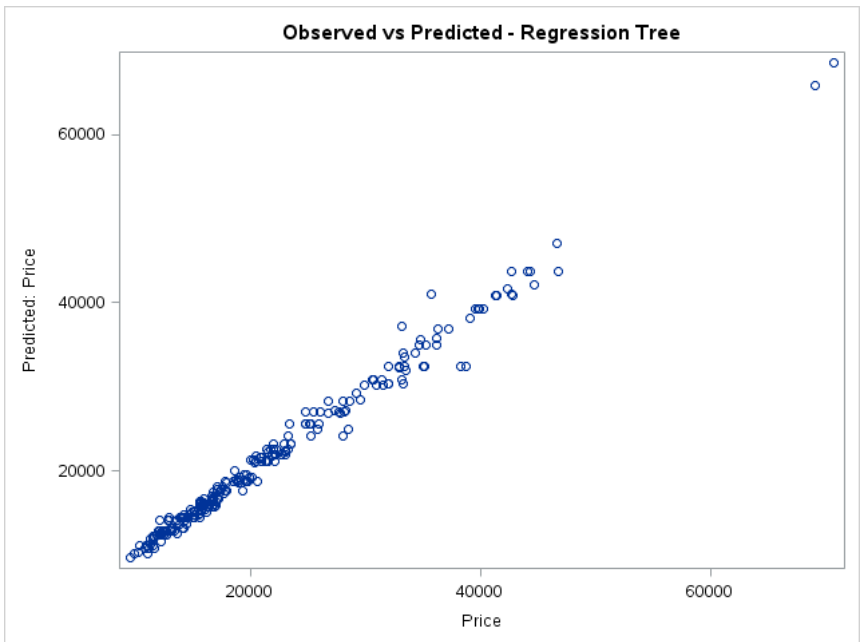

Figure 12: Observed vs Predicted Price - Regression Tree.
The error rates for these models were calculated by using the following formula:

Mean $\left(\sum(\mid\right.$ (observed - predicted $) \mid /$ observed $\left.) * 100\right)$

The results are tabulated below.

Table - 7: Model Error Rates

\begin{tabular}{|l|l|}
\hline Model & Error Rate \\
\hline Lasso Regression & $3.581 \%$ \\
\hline Multiple Regression & $3.468 \%$ \\
\hline Regression Tree & $3.512 \%$ \\
\hline
\end{tabular}

Looking at our models, we see that error rate in multiple regression $(3.468 \%)$ is smaller than the error rate in Regression tree $(3.512 \%)$ which is lesser than the error rate in Lasso Regression (3.581\%).

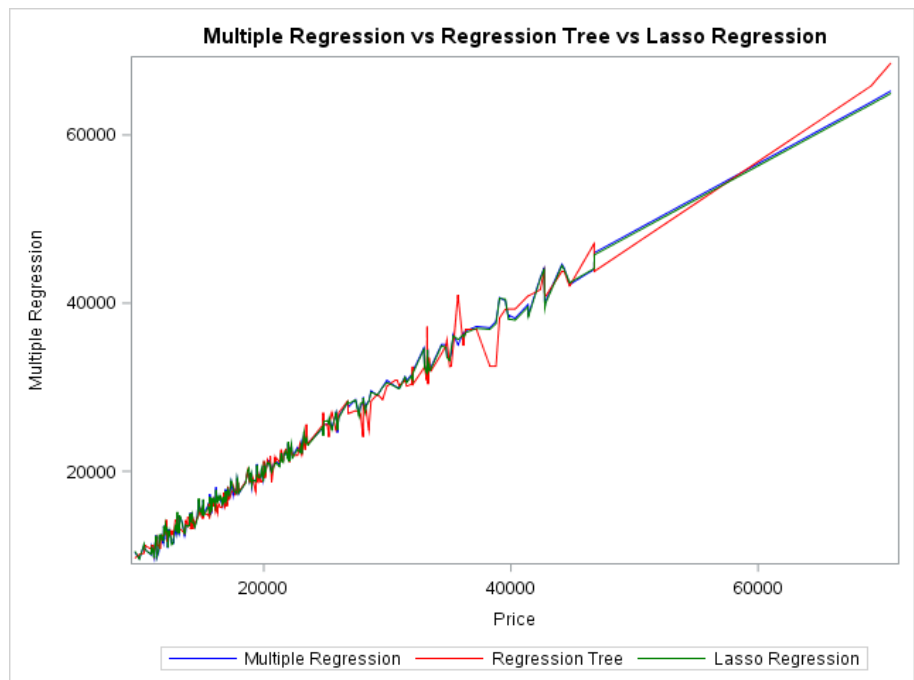

Figure 13: A color-coded line graph comparing the price predictions of the different models with the actual value

However, from this, we can't conclude that our hypothesis holds good since the error rates were found only on one variation of the training and testing data set. By iterating this process[8](with the selection of different records by varying the seed of the random sampling procedure), we will get a set of error rates of lasso regression, multiple regression and regression tree, for the same variation of the data set.

\section{G. Iterative ANOVA based comparison of models}

Using One-way Analysis Of Variance (ANOVA) we need to verify whether the error rates of these models differ significantly from each other.

The process was run 35 times, and the error rates for lasso regression, multiple regression, and regression tree were noted (Table 8) along with the respective seeds of splitting for reproducibility. 
Table - 8: Seed - Error Matrix

\begin{tabular}{|c|c|c|c|}
\hline \multicolumn{2}{|c|}{ Total rows: 35 Total columns: 4} & \multicolumn{2}{|c|}{$1 \leftarrow$ Rows $1.35 \Rightarrow \Rightarrow$} \\
\hline Seed & multiple & tree & lasso \\
\hline 2786 & 3.46839529 & 3.51176948 & 3.58138216 \\
\hline 1589 & 3.6757781 & 3.5680421 & 3.57764978 \\
\hline 100 & 3.67042 & 4.14094084 & 4.01717736 \\
\hline 1458 & 3.49021115 & 3.24932075 & 3.48042261 \\
\hline 2607 & 3.25016677 & 3.91970384 & 3.28921334 \\
\hline 8457 & 3.61699478 & 4.40631117 & 3.71202329 \\
\hline 5841 & 3.44307372 & 3.67629277 & 3.6288628 \\
\hline 6985 & 3.68199985 & 3.77225492 & 3.64147977 \\
\hline 4185 & 3.51752289 & 3.58014847 & 3.45390467 \\
\hline 1208 & 3.58700681 & 4.02278469 & 3.48855108 \\
\hline 7408 & 3.55941912 & 3.48832635 & 3.56545587 \\
\hline 7985 & 3.38059236 & 3.7056248 & 3.33113402 \\
\hline 27 & 3.32998887 & 3.41531015 & 3.24091976 \\
\hline 3451 & 3.64931838 & 3.89251644 & 3.71782049 \\
\hline 8 & 3.6142105 & 4.21475854 & 3.71334439 \\
\hline 587 & $3 \cap ก 37316$ & 3 354583KK & 3105181 \\
\hline
\end{tabular}

This table contains the error rates of the three models for 35 different variations of training and test data.

The data were recoded to perform ANOVA.

Table - 9: Recoded Error Data

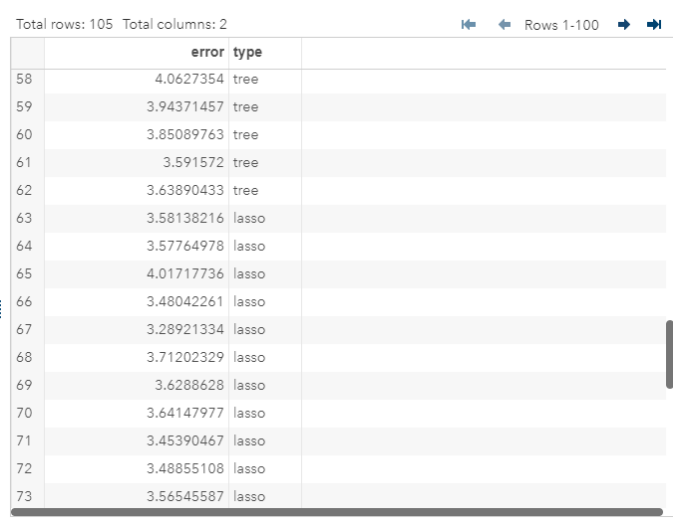

This table contains the recoded error rates.

The ANOVA procedure was carried out, and the results were tabulated in table 10.

\section{Table - 10: ANOVA Summary}
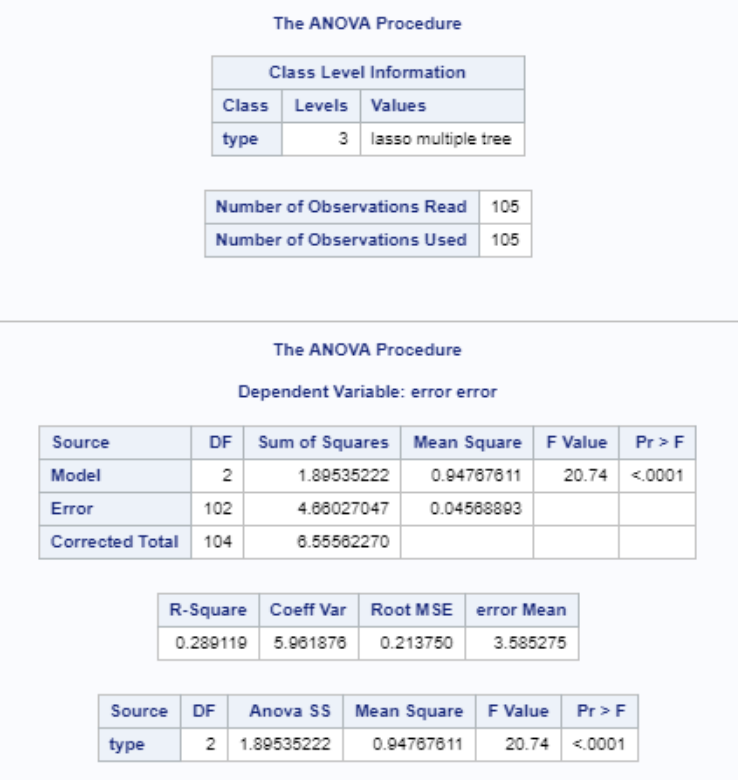

With the P-value being lesser than 0.05 , we can confirm that the error rates are significantly different from each other. Their distribution is also plotted (Fig 14).

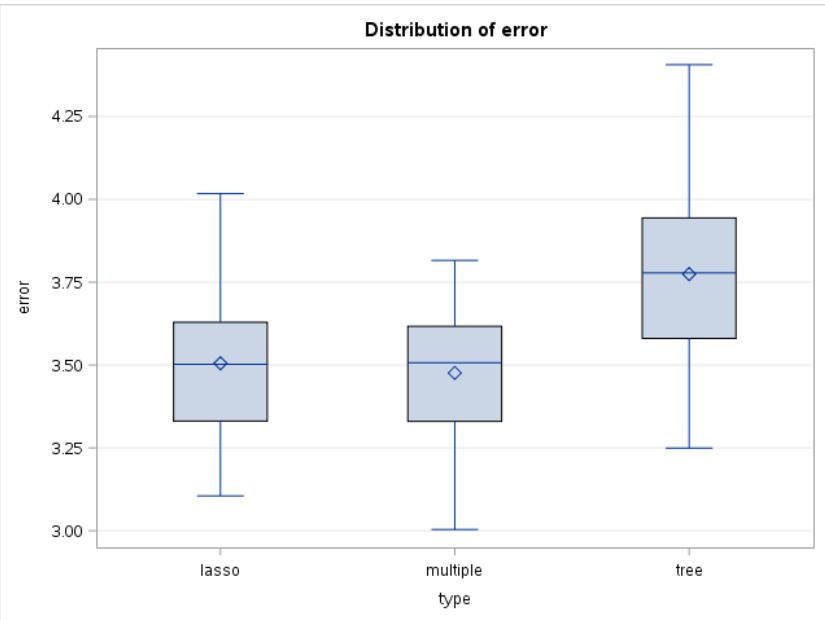

Figure 14: The distribution of the error rates of each model type is represented using box plots.

Table - 11: Mean Error Rates

\begin{tabular}{|c|c|c|c|}
\hline \multirow{2}{*}{$\begin{array}{l}\text { Level of } \\
\text { type }\end{array}$} & \multirow[b]{2}{*}{$\mathbf{N}$} & \multicolumn{2}{|c|}{ error } \\
\hline & & Mean & Std Dev \\
\hline lasso & 35 & 3.50528606 & 0.19866047 \\
\hline multiple & 35 & 3.47601284 & 0.18868152 \\
\hline tree & 35 & 3.77452740 & 0.24825250 \\
\hline
\end{tabular}

The mean error rates of the models (Table 11) might be misleading, since we can't be sure about which groups/models have significantly different means from the other. This is due to the existence of more than 2 groups/levels. One-Way ANOVA can only find out whether there exists any significant difference between any of the groups. To get a clearer picture, we need to perform a post-hoc test to find the groups which have significantly different means.

We are performing a Tukey's test (Tukey's Honest Significant Difference Test) to find out the groups which are actually different from each other.

The test compares all possible pairs of means and checks for statistically significant differences between them. Since the sample size for all the groups is the same, we do not use the Tukey-Kramer Method[7], and use the standard version of the algorithm.

Table - 12: Tukey's Test Summary

\begin{tabular}{|l|r|}
\hline Alpha & 0.05 \\
\hline Error Degrees of Freedom & 102 \\
\hline Error Mean Square & 0.045689 \\
\hline Critical Value of Studentized Range & 3.36358 \\
\hline Minimum Significant Difference & 0.1215 \\
\hline
\end{tabular}

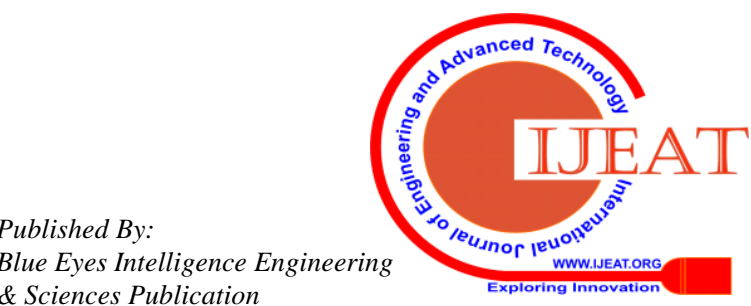




\section{Used Cars Price Prediction using Supervised Learning Techniques}

From table 12 we can get the critical value of the studentized range and the minimum significant difference. The result is plotted graphically (Fig 15).

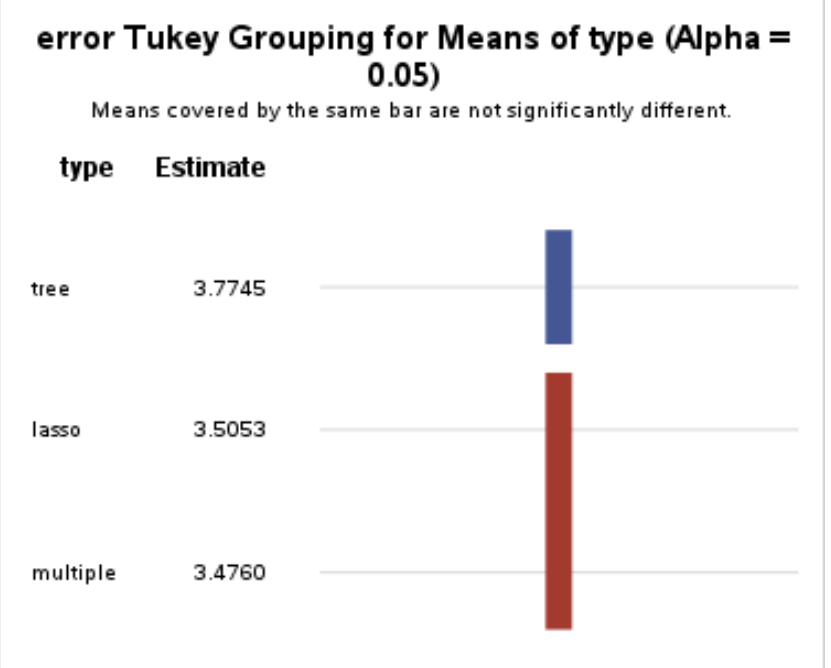

Figure 15: The result of Tukey's Test is plotted in such a way that the means which are covered by the same bar are not significantly different.

From Fig. 15, we can infer that the mean error rates of lasso regression models and multiple regression models are not significantly different, but the mean error rate of regression trees are higher and significantly different from the other two.

\section{CONCLUSION AND FUTURE ENHANCEMENT}

The prediction error rate of all the models was well under the accepted $5 \%$ of error. But, on further analysis, the mean error of the regression tree model was found to be more than the mean error rate of the multiple regression and lasso regression models. Even though for some seeds the regression tree has better accuracy, its error rates are higher for the rest. This has been confirmed by performing an ANOVA. Also, the post-hoc test revealed that the error rates in multiple regression models and lasso regression models aren't significantly different from each other. To get even more accurate models, we can also choose more advanced machine learning algorithms such as random forests, an ensemble learning algorithm which creates multiple decision/regression trees, which brings down overfitting massively or Boosting, which tries to bias the overall model by weighing in the favor of good performers. More data from newer websites and different countries can also be scraped and this data can be used to retrain these models to check for reproducibility.

\section{REFERENCES}

[1] Shonda Kuiper (2008) Introduction to Multiple Regression: How Much Is Your Car Worth?, Journal of Statistics Education, 16:3, DOI: 10.1080/10691898.2008.11889579

[2] Geurts P. (2009) Bias vs Variance Decomposition for Regression and Classification. In: Maimon O., Rokach L. (eds) Data Mining and Knowledge Discovery Handbook. Springer, Boston, MA

[3] Robert T. (1996) Regression Shrinkage and Selection Via the Lasso. In: Journal of the Royal Statistical Society: Series B (Methodological) Volume 58, Issue 1
[4] Hastie, Trevor, and Daryl Pregibon. Shrinking trees. AT \& T Bell Laboratories, 1990.

[5] Kim, Tae Kyun. "Understanding one-way ANOVA using conceptual figures." Korean journal of anesthesiology 70.1 (2017): 22.

[6] Haynes W. (2013) Tukey's Test. In: Dubitzky W., Wolkenhauer O., Cho KH., Yokota H. (eds) Encyclopedia of Systems Biology. Springer, New York, NY

[7] Jaccard, James, Michael A. Becker, and Gregory Wood. "Pairwise multiple comparison procedures: A review." Psychological Bulletin 96.3 (1984): 589

[8] Dupac, Václac, ed. Sampling from a finite population. Marcel Dekker, Incorporated, 1981.

\section{AUTHORS PROFILE}

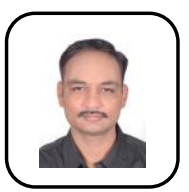

Pattabiraman Venkatasubbu obtained his $\mathrm{Ph} . \mathrm{D}$. from Bharathiar University, India. He has a total Professiona experience of 19 years working in various prestigious institutions. He is currently a Professor at Vellore Institute of Technology, Chennai Campus, India. He has authored several books in the field of Computer Science. He is a Senior member of International Association of Computer Science and Information Technology (IACSIT) also he is member in various professional societies namely ACM, IEEE, ISTE, CSI, Society for Research in Information Security and Privacy- SRISP and Academy \& Industry Research Collaboration Center (AIRCC). Dr. Pattabiraman's teaching and research expertise covers a wide range of subject area including Knowledge discovery and Data mining, Big Data Analytics, Machine Learning, Deep Learning, Database technologies, Data Structures and Analysis of Algorithms etc., He has also received several awards in his career.

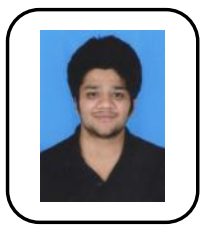

Mukkesh Ganesh is a B.tech Computer Science student at Vellore Institute of Technology, Chennai. A budding $\mathrm{ML}$ and $\mathrm{AI}$ enthusiast, who is working on leveraging the power of AI to solve a variety of problems. He is currently researching on the detection of anomalies using deep learning methods and time series forecasting of kidnapping rates in India. He is also interested in the prospect of enhancing Edge Computing in the field of IOT, to decentralize the heavy server-level processing. His other areas of interest include algorithm design, parallel and distributed computing and theory of computation. He is also working on several projects which span across different fields in Computer Science. He is a finalist of Smart India Hackathon 2019 software edition, and was one of the youngest team leaders in the government organized competition. 